\title{
ON THE SECOND DUAL OF THE LORENTZ SPACE
}

\author{
PRATIBHA G. GHATAGE AND BRIAN M. SCOTT
}

\begin{abstract}
If $\phi(t)=t^{1 / p}(p>1)$ and $(X, S, \mu)$ is a completely nonatomic finite measure space, then the dual of the Lorentz space $N_{\phi}$ is denoted by $M_{\phi}$ and the closure of the simple functions in $M_{\phi}$ by $M_{\phi}^{0}$. It is known that $\left(M_{\phi}^{0}\right)^{*}=N_{\phi}$. In this note we show that given a positive number $\beta<1$ it is possible to construct a set of contractive embeddings of $\left(l_{\infty} / c_{0}\right)^{*}$ into $\left(M_{\phi} / M_{\phi}^{0}\right)^{*}$, each of which is bounded below by $M=M(\beta) \rightarrow 1$ as $\beta \rightarrow 0^{+}$. The union of the ranges of these embeddings is a total set in $\left(M_{\phi} / M_{\phi}^{0}\right)^{*}$.
\end{abstract}

In this note we study the bounded linear functionals on $M_{\phi}$ which annihilate the simple functions in $M_{\phi}$. The dual space of $M_{\phi}^{0}$ (= the closure of the simple functions in $M_{\phi}$ ) has been shown to be isometrically isomorphic to $N_{\phi}$, and thus the present results contribute toward a somewhat better understanding of $M_{\phi}^{*}$.

Notation. Suppose that $(X, S, \mu)$ is a completely nonatomic finite measure space, and $\phi(t)=t^{1 / p}(p>1)$ is a concave function. Then the Lorentz space $N_{\phi}$ is defined by

$$
N_{\phi}=\left\{f: f \text { is measurable \& } \int_{0}^{\infty} \phi\left(\mu\left(F_{y}\right)\right) d y<\infty\right\},
$$

where $F_{y}=\{x \in X:|f(x)|>y\}$;

$$
M_{\phi}=\left\{f: f \text { is measurable \& } \sup _{\mu(E)>0} \frac{1}{\phi(\mu(E))} \int_{E}|f(x)| d \mu<\infty\right\}
$$

and

$$
M_{\phi}^{0}=\left\{f \in M_{\phi}: \lim _{\mu(E) \rightarrow 0} \frac{1}{\phi(\mu(E))} \int_{E}|f(x)| d \mu=0\right\} .
$$

It is known that $N_{\phi}^{*}=M_{\phi}, M_{\phi}^{0}$ is the closure of the simple functions in $M_{\phi}$, and $\left(M_{\phi}^{0}\right)^{*}=N_{\phi}$. For more details see [2 and 3]. Finally, $q$ is defined as usual by $1 / p+1 / q=1$.

REMARK. It is easy to verify that $N_{\phi}$ is an (AL)-space, since the simple functions are dense in $N_{\phi} ; M_{\phi}$ is therefore isometrically isomorphic to $C(S)$, where the compact Hausdorff space $S$ may be taken to be the extreme points of the positive face of the unit sphere of $M_{\phi}^{*}$ with the weak ${ }^{*}$ topology. However, this abstract characterization seems not to help in finding a concrete description of a single element of $\left(M_{\phi} / M_{\phi}^{0}\right)^{*}$. (See $[\mathbf{1}$, Chapter $6, \S \S 1,2]$.) We have instead the following construction.

Fix a positive number $\beta<1$. The nonatomicity of $\mu$ allows us to choose a sequence $\mathcal{E}=\left\{E_{n}\right\}$ of pairwise disjoint, measurable sets with $\mu\left(E_{n}\right)=\beta^{n p}$ (provided,

Received by the editors September 15, 1983.

1980 Mathematics Subject Classification. Primary 46E30.

Key words and phrases. Lorentz space. 
of course, that $\left.\sum_{n=1}^{\infty} \beta^{n p}=\beta^{p} /\left(1-\beta^{p}\right) \leq \mu(X)\right)$. We denote the set of all such sequences by $\mathbf{E}_{\beta}$.

LEMMA 1. For each $\mathcal{E} \in \mathbf{E}_{\beta}$ define $T_{\mathcal{E}}:\left(l_{\infty} / c_{0}\right)^{*} \rightarrow\left(M_{\phi} / M_{\phi}^{0}\right)^{*}$ by $T_{\mathcal{E}}(L)=\Lambda$, where

$$
\Lambda\left(g+M_{\phi}^{0}\right)=L\left\{\frac{1}{\mu\left(E_{n}\right)^{1 / p}} \int_{E_{n}} g d \mu+c_{0}\right\} ;
$$

then $T_{E}$ is a contractive embedding.

Proof. Note first that for each $g \in M_{\phi}$,

$$
\left\|\left\{\frac{1}{\mu\left(E_{n}\right)^{1 / p}} \int_{E_{n}} g d \mu\right\}\right\|_{\infty} \leq\|g\|_{M_{\phi}} .
$$

Moreover,

$$
\left\{\frac{1}{\mu\left(E_{n}\right)^{1 / p}} \int_{E_{n}} g d \mu\right\} \in c_{0} \quad \text { whenever } g \in M_{\phi}^{0} .
$$

We now prove that $T_{\mathcal{E}}$ is bounded below by a number $M(\beta)$ that (i) is independent of $E$, and (ii) approaches 1 as $\beta \rightarrow 0^{+}$.

For any $\left\{r_{n}\right\} \in l_{\infty}, g=\sum\left(r_{n} / \mu\left(E_{n}\right)^{1 / q}\right) \psi_{E_{n}}$ is a well-defined function. To prove that $g \in M_{\phi}$ we need only look at

$$
\frac{1}{\mu(E)^{1 / p}} \int_{E}|g| d \mu \text { for } E \subseteq \bigcup_{n} E_{n} .
$$

If we write $\mu\left(E \cap E_{n}\right)=\alpha_{n} \mu\left(E_{n}\right)$, then $0 \leq \alpha_{n} \leq 1$, and

$$
\begin{aligned}
\frac{1}{\mu(E)^{1 / p}} \int_{E}|g| d \mu & =\frac{1}{\left(\sum \alpha_{n} \beta^{n p}\right)^{1 / p}} \sum \frac{\left|r_{n}\right| \mu\left(E \cap E_{n}\right)}{\mu\left(E_{n}\right)^{1 / q}} \\
& =\frac{1}{\left(\sum \alpha_{n} \beta^{n p}\right)^{1 / p}} \sum\left|r_{n}\right| \alpha_{n} \mu\left(E_{n}\right)^{1 / p} \\
& =\sum \frac{\left|r_{n}\right| \alpha_{n} \beta^{n}}{\left(\sum \alpha_{n} \beta^{n p}\right)^{1 / p}} \leq\left\|\left\{r_{n}\right\}\right\|_{\infty} \frac{\sum \alpha_{n} \beta^{n}}{\left(\sum \alpha_{n} \beta^{n p}\right)^{1 / p}} .
\end{aligned}
$$

Let

$$
M(\beta)=\sup _{\substack{\left\{\alpha_{n}\right\} \\ 0 \leq \alpha_{n} \leq 1}} \frac{\sum \alpha_{n} \beta^{n}}{\left(\sum \alpha_{n} \beta^{n p}\right)^{1 / p}}
$$

Then $\|g\|_{M_{\phi}} \leq M(\beta)\left\|\left\{r_{n}\right\}\right\|_{\infty}$, and the desired result follows from the next lemma.

LEMMA 2. $M(\beta)=\left(1-\beta^{p}\right)^{1 / p} /(1-\beta)$.

PROOF. We start by proving a slightly more general result. Let $\left\{\beta_{n}\right\}$ be a decreasing sequence of positive numbers such that $\sum \beta_{n}<\infty$. Let $D=[0,1]^{\infty} \backslash\{\overrightarrow{0}\}$, where $\overrightarrow{0}=\langle 0,0, \ldots\rangle$. For $\vec{\alpha}=\left\{\alpha_{n}\right\} \in D$ define

$$
f(\vec{\alpha})=\sum \beta_{n} \alpha_{n} /\left(\sum \beta_{n}^{p} \alpha_{n}\right)^{1 / p}
$$

Finally, for $k \geq 1$ define $\overrightarrow{z^{k}} \in D$ by

$$
z_{i}^{k}= \begin{cases}0, & i<k \\ 1, & i \geq k\end{cases}
$$


Our result will follow easily once we establish the following important claim.

Claim 1. $\sup _{\vec{\alpha} \in D} f(\vec{\alpha})=\sup _{k} f\left(\overrightarrow{z^{k}}\right)$.

To prove the claim we need some extra notation. For $\vec{\alpha} \in D$ and $n \geq 1$ let

$$
t_{n}(\vec{\alpha})=\sum_{k=1}^{n} \beta_{k} \alpha_{k} \quad \text { and } \quad b_{n}(\vec{\alpha})=\left(\sum_{k=1}^{n} \beta_{k}^{p} \alpha_{k}\right)^{1 / p}
$$

If $b_{n}(\vec{\alpha}) \neq 0$ let $f_{n}(\vec{\alpha})=t_{n}(\vec{\alpha}) / b_{n}(\vec{\alpha})$; clearly $f_{n}(\vec{\alpha}) \rightarrow f(\vec{\alpha})$ as $n \rightarrow \infty$. It suffices, therefore, to prove for any $\vec{\alpha} \in D$ and $n \geq 1$ that there is a $k \leq n$ such that $f_{n}(\vec{\alpha}) \leq f_{n}\left(\overrightarrow{z^{k}}\right) \leq f\left(\overrightarrow{z^{k}}\right)$. The existence of such a $k$ depends on the following elementary observations.

Fact 1. Suppose $0<b \leq t$ and $\beta>0$, and let $h(x)=(t+\beta x) /\left(b^{p}+\beta^{p} x\right)^{1 / p}$ for $0 \leq x \leq 1$. Then $h(x)$ attains its maximum at $x=0$ or $x=1$. Moreover, $h(x)$ attains its maximum at $x=1$ whenever $\beta^{p-1} \leq b^{p} / t$.

Fact 2. If $0<x<b \leq t$ and $(t+x) /\left(b^{p}+x^{p}\right)^{1 / p}<t / b$, then

$$
(t-x) /\left(b^{p}-x^{p}\right)^{1 / p}>t / b .
$$

Fact 3. If $0<x \leq y<b \leq t$ and $(t+x) /\left(b^{p}+x^{p}\right)^{1 / p}<t / b$, then

$$
(t-y) /\left(b^{p}-y^{p}\right)^{1-p}>t / b .
$$

The proof of Fact 1 is trivial. If the conclusion of Fact 2 fails, then

$$
(1+x / t)^{p}<1+(x / b)^{p} \text { and }(1-x / t)^{p} \leq 1-(x / b)^{p}
$$

so

$$
(1+x / t)^{p}+(1-x / t)^{p}<2 .
$$

But $g(y)=(1+y)^{p}+(1-y)^{p}$ is an increasing function for $0 \leq y \leq 1$, and $g(0)=2$, so, in particular, $g(x / t)>2$; this contradiction establishes the result. Fact 3 generalizes and follows from Fact 2. To see this, note that as a function of $x,(t+x) /\left(b^{p}+x^{p}\right)^{1 / p}$ is increasing if $0 \leq x<\left(b^{p} / t\right)^{1 /(p-1)}$, decreasing if $x>\left(b^{p} / t\right)^{1 /(p-1)}$, and equal to $t / b$ when $x=0$. The hypothesis of Fact 3 therefore implies that $x>\left(b^{p} / t\right)^{1 /(p-1)}$, so the conclusion is immediate from Fact 2.

We can now prove Claim 1. Since

Fix $\vec{\alpha} \in D$ and $n \geq 1$. Let $\overrightarrow{\alpha^{1}} \in D$ be obtained from $\vec{\alpha}$ by changing $\alpha_{n}$ to 1 .

$$
\beta_{n}^{p-1}<\beta_{n-1}^{p-1} \leq \frac{\left(b_{n-1}(\vec{\alpha})\right)^{p}}{t_{n-1}(\vec{\alpha})} \leq 1,
$$

Fact 1 implies that $f_{n}\left(\overrightarrow{\alpha^{1}}\right) \geq f_{n}(\vec{\alpha})$. Now replace $\alpha_{n-1}^{1}$ by 1 or 0 to get $\overrightarrow{\alpha^{2}}$, whichever makes $f_{n}\left(\overrightarrow{\alpha^{2}}\right)$ larger; Fact 1 shows that $f_{n}\left(\overrightarrow{\alpha^{2}}\right) \geq f_{n}\left(\overrightarrow{\alpha^{1}}\right)$. Continue in this way to obtain $\overrightarrow{\alpha^{n}} \in D$ such that $f_{n}\left(\overrightarrow{\alpha^{n}}\right) \geq f_{n}(\vec{\alpha}), \alpha_{1}^{n}, \alpha_{2}^{n}, \ldots, \alpha_{n}^{n} \in\{0,1\}$, and $\alpha_{n}^{n}=1$.

Suppose there are $m$ and $l$ such that $1 \leq m<l<n, \alpha_{m}^{n}=1, \alpha_{l}^{n}=0$, and $\alpha_{l+1}^{n}=\cdots=\alpha_{n}^{n}=1$. Let $\vec{u}$ be obtained from $\overrightarrow{\alpha^{n}}$ by replacing $\alpha_{m}^{n}$ by 0 , let $\vec{v}$ be obtained from $\overrightarrow{\alpha^{n}}$ by replacing $\alpha_{l}^{n}$ by 1 , and assume that $f_{n}\left(\overrightarrow{\alpha^{n}}\right)>f_{n}(\vec{v})$. 
It follows from Fact 3 that $f_{n}(\vec{u})>f_{n}\left(\overrightarrow{\alpha^{n}}\right)$ : just set $t=t_{n}\left(\overrightarrow{\alpha^{n}}\right), b=b_{n}\left(\overrightarrow{\alpha^{n}}\right)$, $x=\beta_{l}$, and $y=\beta_{m}$. In short, we may replace $\overrightarrow{\alpha^{n}}$ by either $\vec{u}$ or $\vec{v}$ without decreasing the value of $f_{n}$. It now follows by an easy finite induction that there is some $k \leq l$ such that $f_{n}\left(\overrightarrow{\alpha^{n}}\right) \leq f_{n}\left(\overrightarrow{z^{k}}\right)$; and repeated applications of Fact 1 , $f_{n}\left(\overrightarrow{z^{k}}\right) \leq f_{n+1}\left(\overrightarrow{z^{k}}\right) \leq f_{n+2}\left(\overrightarrow{z^{k}}\right) \leq \cdots$, whence $f_{n}\left(\overrightarrow{z^{k}}\right) \leq f\left(\overrightarrow{z^{k}}\right)$, and the proof of Claim 1 is complete.

To finish the proof of Lemma 2 , just note that if $0<\beta<1$ and $\beta_{n}=\beta^{n}$ for $n \geq 1$, then

$$
f\left(\overrightarrow{z^{k}}\right)=\frac{\sum_{n=k}^{\infty} \beta^{n}}{\left(\sum_{n=k}^{\infty} \beta^{n p}\right)^{1 / p}}=\frac{\beta^{k} /(1-\beta)}{\left[\beta^{k p} /\left(1-\beta^{p}\right)\right]^{1 / p}}=\frac{\left(1-\beta^{p}\right)^{1 / p}}{1-\beta},
$$

and hence,

$$
\sup _{\vec{\alpha} \in D} f(\vec{\alpha})=\frac{\left(1-\beta^{p}\right)^{1 / p}}{1-\beta} .
$$

This is an increasing function of $\beta$, so for any $M>1$ we can choose $\beta$ to make $M(\beta)=M$. This completes the proofs of Lemmas 1 and 2 .

LEMmA 3. For any $M>1$ there is a $\beta, 0<\beta<1$, such that the family $\left\{T_{\mathcal{E}}: \mathcal{E} \in \mathbf{E}_{\beta}\right\}$ of embeddings of $\left(l_{\infty} / c_{0}\right)^{*}$ into $\left(M_{\phi} / M_{\phi}^{0}\right)^{*}$ satisfies the following condition: If $L \in\left(l_{\infty} / c_{0}\right)^{*}, \mathcal{E} \in \mathbf{E}_{\beta}$, and $\Lambda=T_{\mathcal{E}}(L)$, then $\|\Lambda\| \leq\|L\| \leq M\|\Lambda\|$.

ProOF. Note that

$$
\left\{r_{n}\right\} \mapsto g=\sum_{n=1}^{\infty} \frac{r_{n}}{\mu\left(E_{n}\right)^{1 / q}} \chi_{E_{n}}
$$

defines a bounded map from $l_{\infty}$ into $M_{\phi}$. Moreover, if $r_{n} \rightarrow 0$, then

$$
g_{n}=\sum_{k=1}^{n} \frac{r_{k}}{\mu\left(E_{k}\right)^{1 / q}} \chi_{E_{k}} \rightarrow g \text { in the } M_{\phi} \text {-norm, }
$$

since $\left\|g-g_{n}\right\|_{M_{\phi}} \leq M\left\|\left\{r_{k}\right\}_{k=n}^{\infty}\right\|_{\infty}$; thus, $g \in M_{\phi}^{0}$. (Of course, $\beta$ is chosen so that $M(\beta)=M$.) Hence the map

$$
j_{\mathcal{E}}: l_{\infty} / c_{0} \rightarrow M_{\phi} / M_{\phi}^{0}:\left\{r_{n}\right\}+c_{0} \mapsto g+M_{\phi}^{0}
$$

is well defined and $\left\|j_{\mathcal{E}}\right\| \leq M$. Also, if $j_{\mathcal{E}}\left(\left\{r_{n}\right\}\right)=g$, then

$$
\frac{1}{\mu\left(E_{n}\right)^{1 / q}} \int_{E_{n}} g d \mu=r_{n}
$$

thus, the adjoint $j_{\mathcal{E}}^{*}:\left(M_{\phi} / M_{\phi}^{0}\right)^{*} \rightarrow\left(l_{\infty} / c_{0}\right)^{*}$ satisfies $j_{\mathcal{E}}^{*} \circ T_{\mathcal{E}}=$ id and $\left\|j_{\mathcal{E}}^{*}\right\| \leq M$. This completes the proof of Lemma 3.

We now prove a result about elements of $M_{\phi} \backslash M_{\phi}^{0}$; this will help us prove that the union of the ranges of all the $T_{\mathcal{E}}\left(\mathcal{E} \in \mathbf{E}_{\beta}\right)$ is a total set in $\left(M_{\phi} / M_{\phi}^{0}\right)^{*}$.

LEMMA 4. If $g \in M_{\phi} \backslash M_{\phi}^{0}$, there is a sequence $\left\{A_{n}\right\}$ of pairwise disjoint, measurable sets such that $\mu\left(A_{n}\right) \rightarrow 0$, but

$$
\inf _{n \geq 1} \frac{1}{\mu\left(A_{n}\right)^{1 / p}} \int_{A_{n}}|g| d \mu>0 .
$$


ProOF. Recall that

$$
M_{\phi}^{0}=\left\{g \in M_{\phi}: \lim _{\mu(E) \rightarrow 0} \frac{1}{\mu(E)^{1 / p}} \int_{E}|g| d \mu=0\right\} .
$$

Thus if $g \notin M_{\phi}^{0}$, we may assume $g \geq 0$ and there is a sequence $\left\{A_{n}\right\}$ of measurable sets of pairwise measure such that if $\alpha_{n}=\mu\left(A_{n}\right)$, then $\lim _{n} \alpha_{n}=0$, but $\inf _{n} a_{n}=$ $\varepsilon>0$, where $a_{n}=\alpha_{n}^{-1 / p} \int_{A_{n}} g d \mu$.

Let

$$
\begin{gathered}
A_{n, m}=A_{n} \backslash \bigcup_{k \geq m} A_{k}, \quad \alpha_{n, m}=\mu\left(A_{n, m}\right), \quad a_{n, m}=\frac{1}{\alpha_{n, m}^{1 / p}} \int_{A_{n, m}} g d \mu \\
B_{n, m}=A_{n} \backslash A_{n, m}, \quad \beta_{n, m}=\mu\left(B_{n, m}\right), \quad \text { and } \quad b_{n, m}=\frac{1}{\beta_{n, m}^{1 / p}} \int_{B_{n, m}} g d \mu .
\end{gathered}
$$

Then

$$
\begin{aligned}
\varepsilon & \leq a_{n}=\alpha_{n}^{-1 / p}\left(\int_{A_{n, m}} g d \mu+\int_{B_{n, m}} g d \mu\right) \\
& =\alpha_{n}^{-1 / p}\left(\alpha_{n, m}^{1 / p} a_{n, m}+\beta_{n, m}^{1 / p} b_{n, m}\right) \leq \alpha_{n}^{-1 / p}\left(\alpha_{n, m}^{1 / p} a_{n, m}+\beta_{n, m}^{1 / p}\|g\|_{M_{\phi}}\right) .
\end{aligned}
$$

Hence,

$$
\alpha_{n}^{1 / p} \varepsilon-\beta_{n, m}^{1 / p}\|g\|_{M_{\phi}} \leq \alpha_{n, m}^{1 / p} a_{n, m} \quad \text { or } \quad a_{n, m} \geq\left(\frac{\alpha_{n}}{\alpha_{n, m}}\right)^{1 / p} \varepsilon-\left(\frac{\beta_{n, m}}{\alpha_{n, m}}\right)^{1 / p}\|g\|_{M_{\phi}} .
$$

By passing, if necessary, to a subsequence, we may assume $\sum \alpha_{n}<\infty$, in which case $\lim _{m}\left(\beta_{n, m} / \alpha_{n, m}\right)=0$, so $a_{n, m} \geq \varepsilon / 2$ for sufficiently large $m$ (and a fixed $n$ ).

For each $n$ let $m(n)$ be such an $m$. Let $A_{1}^{\prime}=A_{1, m(1)}$ and $n_{1}=1$. Given $n_{k}$, let $A_{k}^{\prime}=A_{n_{k}, m\left(n_{k}\right)}$ and $n_{k+1}=m\left(n_{k}\right)$. Let $\alpha_{k}^{\prime}=\mu\left(A_{k}^{\prime}\right)$ and $a_{k}^{\prime}=\alpha_{k}^{-1 / p} \int_{A_{k}^{\prime}} g d \mu$. Clearly $\lim _{k} \alpha_{k}^{\prime}=0, \inf _{k} a_{k}^{\prime} \geq \varepsilon / 2$, and the sets $A_{k}^{\prime}$ are pairwise disjoint.

THEOREM 1. The subset of $\left(M_{\phi} / M_{\phi}^{0}\right)^{*}$ consisting of the union of the ranges of all the embeddings $T_{\mathcal{E}}\left(\mathcal{E} \in \mathbf{E}_{\beta}\right)$ is total.

Proof. Suppose $g \in M_{\phi}$ is such that whenever $L \in l_{\infty}^{*} \cap c_{0}^{\perp}, \mathcal{E} \in \mathbf{E}_{\beta}$, and $\Lambda=T_{\mathcal{E}}(L)$, then $\Lambda(g)=0$. We show that $g \in M_{\phi}^{0}$.

Suppose not. Without loss of generality we may assume $g \geq 0$. By Lemma 4 there is a sequence $\left\{A_{n}\right\}$ of pairwise disjoint sets of positive measure such that if $\alpha_{n}=\mu\left(A_{n}\right)$ and $a_{n}=\alpha_{n}^{-1 / p} \int_{A_{n}} g d \mu$, then $\lim _{n} \alpha_{n}=0$ and $\inf _{n} a_{n}=\varepsilon>0$. Clearly, we may further assume that $\alpha_{1}>\alpha_{2}>\cdots$, and indeed that for each $n$ there is a $k(n)$ such that (i) $\beta^{p k(n)}>\alpha_{n}>\beta^{p(k(n)+1)}$, and (ii) $n<m$ implies $k(n)<k(m)$. (Pass to a subsequence if necessary.) Once again because $\mu$ is completely nonatomic we may choose sets $B_{n}$, disjoint from one another and from the $A_{n}$ 's so that $\mu\left(B_{n}\right)=\beta^{p k(n)}-\alpha_{n}$; let $E_{n}=A_{n} \cup B_{n}$. Then $\mu\left(E_{n}\right)=\beta^{p k(n)}$, and $\left\{E_{n}\right\}$ is a subsequence of some $\mathcal{E} \in \mathbf{E}_{\beta}$. Moreover, if $r_{n}=\beta^{-k(n)} \int_{E_{n}} g d \mu$, then $\left\{r_{n}\right\} \in l_{\infty}$. We now claim that $\left\{r_{n}\right\} \in c_{0}$.

By hypothesis, $L \in l_{\infty}^{*} \cap c_{0}^{\perp}$ implies $T(L)(g)=0$ and, hence, $L\left\{s_{n}\right\}=0$ for some sequence $\left\{s_{n}\right\}$ of which $\left\{r_{n}\right\}$ is a subsequence. But then $\left\{s_{n}\right\} \in c_{0}$, so $\left\{r_{n}\right\} \in c_{0}$ also. 
But

$$
\begin{aligned}
\beta^{-k(n)} \int_{E_{n}} g d \mu & =\beta^{-k(n)}\left[\int_{A_{n}} g d \mu+\int_{B_{n}} g d \mu\right]=\beta^{-k(n)}\left[\alpha_{n}^{1 / p} a_{n}+\int_{B_{n}} g d \mu\right] \\
& \geq \beta^{-k(n)} \alpha_{n}^{1 / p} a_{n}>\frac{\beta^{k(n)+1}}{\beta^{k(n)}} a_{n} \geq \beta \varepsilon
\end{aligned}
$$

so $\left\{r_{n}\right\} \notin c_{0}$. This contradiction shows that $g \in M_{\phi}^{0}$ and completes the proof of Theorem 1 .

Theorem 1 leaves open the question of how much of $\left(M_{\phi} / M_{\phi}^{0}\right)^{*}$ can be filled up with the ranges of the embeddings $T_{\mathcal{E}}$; we do not know the answer even if $\beta$ is allowed to range over $(0,1)$. For a fixed $\beta$, at least, we suspect there are bounded linear functionals on $M_{\phi} / M_{\phi}^{0}$ that do not arise as linear combinations of functionals $T_{\mathcal{E}}(L), \mathcal{E} \in \mathbf{E}_{\beta}$ and $L \in\left(l_{\infty} / c_{0}\right)^{*}$.

We do know that if $\mathcal{E}=\left\{E_{n}\right\}$ and $\mathcal{F}=\left\{F_{n}\right\}$ in $\mathbf{E}_{\beta}$ are eventually disjoint $\left(\bigcup_{n=k}^{\infty} E_{n} \cap \bigcup_{n=k}^{\infty} F_{n}=\varnothing\right.$ for some $\left.k \geq 1\right)$, then the ranges of $T_{\mathcal{E}}$ and $T_{\mathcal{F}}$ make a positive angle (depending on $\beta$ but not on $\mathcal{E}$ and $\mathcal{F}$ ) with each other. (In fact, a somewhat weaker hypothesis will suffice.) The idea is to construct a positive $g \in M_{\phi}$ that is annihilated by every functional in the range of $T_{\mathcal{F}}$ (because $\left.\left\{\mu\left(F_{n}\right)^{-1 / p} \int_{F_{n}} g d \mu\right\} \in c_{0}\right)$ but is such that $\left\{\mu\left(E_{n}\right)^{-1 / p} \int_{E_{n}} g d \mu\right\}$ converges to 1 .

\section{REFERENCES}

1. M. M. Day, Normed linear spaces, Springer-Verlag, New York, 1973.

2. P. Ghatage, The Lorentz space as a dual space, preprint.

3. M. S. Steigerwalt and A. J. White, Some function spaces related to $L_{p}$ spaces, Proc. London Math. Soc. (3) 22 (1971), 137-163.

Department of Mathematics, Cleveland State University, Cleveland, Ohio 44115 